\title{
SOME EXCEPTIONAL PHENOMENA IN MULTIFRACTAL FORMALISM: PART II*
}

\author{
DE-JUN FENG ${ }^{\dagger}$, KA-SING LAU ${ }^{\ddagger}$, AND XIANG-YANG WANG§
}

\begin{abstract}
In Part I we showed that the $L^{q}$-spectrum of the 3 -fold convolution of the Cantor measure has a non-differentiable point at a $q_{0}<0$ [LW], therefore the standard multifractal formalism does not hold. In this Part II, we prove a modified multifractal formalism for the measure.
\end{abstract}

Key words. Cantor measure, Hausdorff dimension, $L^{q}$-spectrum, multifractal formalism

AMS subject classifications. Primary 28A80; Secondary 42B10

1. Introduction. The present paper is a continuation of the work in [LW] for investigating the multifractal structure of the measure $\mu$ of the 3 -fold convolution of the Cantor measure. We first recall some basic setting in [LW]: the probability measure $\mu$ satisfies the self-similar identity

$$
\mu=\sum_{j=0}^{3} p_{j} \mu \circ S_{j}^{-1}
$$

where $S_{j}(x)=\frac{1}{3}(x+2 j)$ for $j \in\{0,1,2,3\}$ and $\left[p_{0}, p_{1}, p_{2}, p_{3}\right]=[1 / 8,3 / 8,3 / 8,1 / 8]$. This is one of the simplest examples of the IFS with overlaps having some exceptional multifractal properties. We can express it in a vector-valued form

$$
\boldsymbol{\mu}(\cdot)=\sum_{j=0}^{2} T_{j} \boldsymbol{\mu}(3 \cdot-j)
$$

where

$$
\boldsymbol{\mu}(A)=\left[\begin{array}{c}
\mu(A \cap[0,1]) \\
\mu((A \cap[0,1])+1) \\
\mu((A \cap[0,1])+2)
\end{array}\right]
$$

for any Borel subset $A \subset \mathbb{R}$, and the matrix-valued coefficients $T_{j}$ are defined by

$$
T_{0}=\left[\begin{array}{ccc}
p_{0} & 0 & 0 \\
0 & p_{1} & 0 \\
p_{3} & 0 & p_{2}
\end{array}\right], T_{1}=\left[\begin{array}{ccc}
0 & p_{0} & 0 \\
p_{2} & 0 & p_{1} \\
0 & p_{3} & 0
\end{array}\right], T_{2}=\left[\begin{array}{ccc}
p_{1} & 0 & p_{0} \\
0 & p_{2} & 0 \\
0 & 0 & p_{3}
\end{array}\right] .
$$

\footnotetext{
*Received May 21, 2005; accepted for publication October 13, 2005.

$\dagger$ Department of Mathematical Sciences, Tsinghua University, Beijing, 100084, P.R. China; and Department of Mathematics, the Chinese University of Hong Kong, Hong Kong (dfeng@ math.tsinghua.edu.cn). The first author was partially supported by the Direct Grant in CUHK and the Natural Science Foundation of China (Grant 10571100).

${ }^{\ddagger}$ Department of Mathematics, the Chinese University of Hong Kong, Hong Kong (kslau@math. cuhk.edu.hk). The second author is partially supported by a HK RGC grant.

$\S$ The communication author. School of Mathematics and Computational Science, Zhong-Shan University, Guang-Zhou, 510275, P. R. China; and Department of Mathematics, the Chinese University of Hong Kong, Hong Kong (mcswxy@zsu.edu.cn). The third author is partially supported by the Postdoctoral Fellow of CUHK and by SRF for ROCS, SEM.
} 
The $L^{q}$-spectrum $\tau(q)$ of $\mu$ has been shown in [LW] to be

$$
\tau(q)=-\lim _{n \rightarrow \infty} \frac{1}{n}\left(\log _{3} \sum_{i=0}^{2} \sum_{|J|=n}\left(\mathbf{e}_{i} T_{J} \mathbf{1}\right)^{q}\right),
$$

where $T_{J}$ denotes $T_{j_{1}} \cdots T_{j_{n}}$ for $J=j_{1} \cdots j_{n}$, and $\mathbf{e}_{i}$ denotes the 3 -dimensional unit vector whose $(i+1)$-th entry is 1 , and 1 the 3 -dimensional vector in which each entry is 1 .

Now we define a sequence of functions $s_{n}(q)$ of $q$ on $\mathbb{R}$ by setting $s_{0}(q)=p_{1}^{q}+p_{2}^{q}$, $s_{1}(q)=\left(p_{0} p_{2}+p_{1} p_{3}\right)^{q}$ and

$$
s_{n}(q)=\sum_{J \in\{0,2\}^{n-1}}\left(\left[p_{2}, p_{1}\right] \bar{T}_{J}\left[\begin{array}{l}
p_{0} \\
p_{3}
\end{array}\right]\right)^{q}, \quad n \geq 2,
$$

where

$$
\bar{T}_{0}=\left[\begin{array}{cc}
p_{0} & 0 \\
p_{3} & p_{2}
\end{array}\right], \quad \bar{T}_{2}=\left[\begin{array}{cc}
p_{1} & p_{0} \\
0 & p_{3}
\end{array}\right] .
$$

It was shown in $[\mathrm{LW}]$ that

TheOREM 1.1. The $L^{q}$-spectrum $\tau(q)$ of $\mu$ is given by

$$
\tau(q)=\left\{\begin{array}{lll}
\log _{3} r(q) & \text { if } & q \geq q_{0}, \\
q \log _{3} 8 & \text { if } \quad q<q_{0},
\end{array}\right.
$$

where $r(q)$ satisfies $\sum_{k=0}^{\infty} s_{k}(q) r(q)^{k+1}=1$ and $q_{0}$ satisfies $8^{-q_{0}} r\left(q_{0}\right)=1 \quad\left(q_{0} \approx\right.$ -1.149). Furthermore $\tau(q)$ is real analytic except for $q=q_{0}$, which is a nondifferentiable point of $\tau$.

For $x \in \operatorname{supp}(\mu)$, let $\alpha(x)=\lim _{\delta \rightarrow 0} \frac{\log \mu\left(B_{\delta}(x)\right)}{\log \delta}$ be the local dimension of $\mu$ at $x$ (if the limit exists). It was proved in [HL] that

TheOrem 1.2. Let $E$ be the set of local dimensions of $\mu$, i.e.,

$$
E=\{\alpha: \alpha=\alpha(x) \text { for some } x \in \operatorname{supp} \mu\} .
$$

Then $E=[\underline{\alpha}, \tilde{\alpha}] \cup\{\bar{\alpha}\}$ with

$$
\underline{\alpha}=\log _{3}(8 / 3), \quad \tilde{\alpha}=\log _{3}\left(8 / \sqrt{\lambda}_{1}\right) \quad \text { and } \quad \bar{\alpha}=\log _{3} 8,
$$

where $\lambda_{1}=(7+\sqrt{13}) / 2$.

As in the above two theorems, the multifractal formalism breaks down resulting from the non-differentiable point of the $L^{q}$-spectrum at $q_{0}$, and the isolated point $\bar{\alpha}$ in the dimension spectrum. It is known in [HL] that the isolated point $\bar{\alpha}$ comes from the two end points of supp $\mu(=[0,3])$. In this Part II, we will overcome these problems by restricting $\mu$ to the interior of its support $[0,3]$. More precisely, we let $\mu_{m}=\left.\mu\right|_{\left[3^{-m}, 3-3^{-m}\right]}$, the restriction of $\mu$ on the interval $\left[3^{-m}, 3-3^{-m}\right]$. Our main theorem is:

TheOREM 1.3. Let $r(q)$ be defined as in Theorem 1.1. Then $r(q)$ is real analytic on $\mathbb{R}$. The $L^{q}$-spectrum of $\mu_{m}$ are the same for all $m \in \mathbb{N}$ and the common value is given by $\tilde{\tau}(q)=\log _{3} r(q), q \in \mathbb{R}$. 
Moreover if we denote $K(\alpha)=\{x \in \operatorname{supp} \mu: \alpha(x)=\alpha\}$, then

$$
\operatorname{dim}_{H} K(\alpha)=\tilde{\tau}^{*}(\alpha), \quad \forall \alpha \in(\underline{\alpha}, \tilde{\alpha}),
$$

where $\tilde{\tau}^{*}(\alpha)$ is the Legendre transform of $\tilde{\tau}(q)$, i.e., $\tilde{\tau}^{*}(\alpha)=\inf \{\alpha q-\tilde{\tau}(q): q \in \mathbb{R}\}$.

For the first part of the theorem, the main task is to show that $r(q)$ is real analytic. Note that this has been proved for $q>q_{0}$ (in fact for $q>-2$ ) in Part I. For the more general $q<0$, it requires more techniques in manipulating the product of the matrices involved in $s_{n}(q)$. It will be discussed in detail in Section 2.

The second part of the theorem follows easily once we have shown that the multifractal formalism holds for $\mu_{0}$. To achieve this point, we represent $\mu_{0}$ as a self-similar measure generated by an IFS with infinitely many similitudes $f_{j}$ :

$$
\mu_{0}=\sum_{j=1}^{\infty} w_{j} \mu_{0} \circ f_{j}^{-1},
$$

where $\left\{w_{j}\right\}_{j=1}^{\infty}$ is a set of probability weights, and the family of $\left\{f_{j}\right\}_{j=1}^{\infty}$ satisfies the following separation condition:

$$
f_{i}(I) \cap f_{j}(I)=\emptyset, \quad i \neq j
$$

where $I=[1,2]$.

Using the representation (1.3) of $\mu_{0}$, we can verify the multifractal formalism for $\mu_{0}$ (actually for a more general self-similar measure generated by a non-overlapping IFS with infinitely many similitudes). We remark that a multifractal analysis for such infinite IFS have been given by Riedi and Mandelbrot [RM], however they need more restrictions on the contraction ratios and their theorem is not applicable here.

We point that a representation similar to (1.3) was set up earlier by Feng in $[\mathrm{F}]$ for the Bernoulli convolutions associated with the golden ratio and some other Pisot numbers. It was shown in $[\mathrm{F}]$ that, in the golden ratio case, the $L^{q}$-spectrum also has a non-differential point in $(-\infty, 0)$; however Feng and Olivier [FO] showed that in this case, the multifractal formalism still holds in the sense that the dimension spectrum and the $L^{q}$-spectrum strictly form a Legendre transform pair.

In the appendix part we show that Theorem 1.2 can also be derived from Theorem 1.3. The argument is considerably simpler than the original combinatorial proof given in [HL]. Actually in the appendix we will provide another one proof depending directly on the estimates of the product of matrices developed here.

2. The $L^{q}$-spectrum. In this section, we prove the first part of Theorem 1.3. As in Part I, we let

$$
M_{0}=8 \bar{T}_{0}=\left[\begin{array}{ll}
1 & 0 \\
1 & 3
\end{array}\right], \quad M_{2}=8 \bar{T}_{2}=\left[\begin{array}{ll}
3 & 1 \\
0 & 1
\end{array}\right] .
$$

For a $2 \times 2$ nonnegative matrix $M$, we let $\|M\|=[1,1] M\left[\begin{array}{l}1 \\ 1\end{array}\right]$.

Note that for any $m>0$ and $J \in\{0,2\}^{m}, J$ can be written as $0^{n_{1}} 2^{n_{2}} \cdots \epsilon^{n_{k}}$ or $2^{n_{1}} 0^{n_{2}} \cdots(2-\epsilon)^{n_{k}}$ for some positive integers $k$ and $n_{1}, \ldots, n_{k}$, where $\varepsilon=0$ or 2 according to $k$ is odd or even. Accordingly, $M_{J}$ can be written as $M_{0}^{n_{1}} M_{2}^{n_{2}} \cdots M_{\varepsilon}^{n_{k}}$ or $M_{2}^{n_{1}} M_{0}^{n_{2}} \cdots M_{2-\varepsilon}^{n_{k}}$.

To evaluate the norm of the product of matrices, for any $k \geq 1$, let $\varepsilon=0$ or 2 according to $k$ is odd or even, and let $n_{1}, \cdots, n_{k} \in \mathbb{N}$. Define

$$
c\left(n_{1}, \cdots, n_{k}\right)=\left\|M_{0}^{n_{1}} M_{2}^{n_{2}} \cdots M_{\varepsilon}^{n_{k}}\right\|\left(=\left\|M_{2}^{n_{1}} M_{0}^{n_{2}} \cdots M_{2-\varepsilon}^{n_{k}}\right\|\right),
$$


and let

$$
\bar{c}\left(n_{1}, \cdots, n_{k}\right)=[0,1] M_{0}^{n_{1}} M_{2}^{n_{2}} \cdots M_{\varepsilon}^{n_{k}}\left[\begin{array}{l}
0 \\
1
\end{array}\right] .
$$

It is clear that $\bar{c}\left(n_{1}, \cdots, n_{k}\right)$ is the $(2,2)$-entry of the matrix $M_{0}^{n_{1}} M_{2}^{n_{2}} \cdots M_{\varepsilon}^{n_{k}}$.

Lemma 2.1. For any $k, l \geq 1, n_{1}, \cdots, n_{k+l} \geq 1$, we have

$$
\begin{gathered}
\bar{c}\left(n_{1}, \cdots, n_{k}\right) \bar{c}\left(n_{k+1}, \cdots, n_{k+l}\right) \leq \bar{c}\left(n_{1}, \cdots, n_{k+l}\right), \\
1 / 8 \leq \bar{c}\left(n_{1}, \cdots, n_{k}\right) / c\left(n_{1}, \cdots, n_{k}\right) \leq 1,
\end{gathered}
$$

and

$$
3^{-k} \leq \bar{c}\left(n_{1}, \cdots, n_{k}\right) / 3^{n_{1}+\cdots+n_{k}} \leq 2^{k} .
$$

Proof. The inequality (2.1) is true for the product of any two nonnegative matrices. For (2.2), it suffices to prove the first inequality. We write $M_{0}^{n_{1}} M_{2}^{n_{2}} \cdots M_{\varepsilon}^{n_{k}}=\left[\begin{array}{cc}\alpha & \beta \\ \gamma & \delta\end{array}\right]$ and $M_{0}^{n_{1}-1} M_{2}^{n_{2}} \cdots M_{\varepsilon}^{n_{k}}=\left[\begin{array}{cc}a & b \\ c & d\end{array}\right]$, then

$$
\left[\begin{array}{ll}
\alpha & \beta \\
\gamma & \delta
\end{array}\right]=\left[\begin{array}{ll}
1 & 0 \\
1 & 3
\end{array}\right]\left[\begin{array}{ll}
a & b \\
c & d
\end{array}\right]=\left[\begin{array}{cc}
a & b \\
a+3 c & b+3 d
\end{array}\right]
$$

It follows that $\alpha \leq \gamma, \beta \leq \delta$. We claim that $\gamma \leq 3 \delta$; this will imply that $(\alpha+\beta+\gamma+$ $\delta) / 8 \leq \delta$ and $(2.2)$ follows.

To prove the claim, we write $M_{0}^{n_{1}} \cdots M_{2-\epsilon}^{n_{k-1}}=\left[\begin{array}{ll}a^{\prime} & b^{\prime} \\ c^{\prime} & d^{\prime}\end{array}\right]$. Note that $M_{0}^{n}=$ $\left[\begin{array}{cc}3^{1}-1 & 0 \\ \frac{3^{n}}{2} & 3^{n}\end{array}\right]$ and $M_{2}^{n}=\left[\begin{array}{cc}3^{n} & \frac{3^{n}-1}{2} \\ 0 & 1\end{array}\right]$. Hence if $k$ is even, then

$$
\left[\begin{array}{ll}
\alpha & \beta \\
\gamma & \delta
\end{array}\right]=\left[\begin{array}{ll}
a^{\prime} & b^{\prime} \\
c^{\prime} & d^{\prime}
\end{array}\right]\left[\begin{array}{cc}
3^{n_{k}} & \frac{3^{n_{k}-1}}{2} \\
0 & 1
\end{array}\right]=\left[\begin{array}{cc}
* & * \\
3^{n_{k}} c^{\prime} & \frac{3^{n_{k}}-1}{2} c^{\prime}+d^{\prime}
\end{array}\right],
$$

so that $\gamma \leq 3 \delta$. Here and afterwards, we use $*$ to represent an entry of a matrix without giving its exact value. If $k$ is odd, then

$$
\left[\begin{array}{ll}
\alpha & \beta \\
\gamma & \delta
\end{array}\right]=\left[\begin{array}{ll}
a^{\prime} & b^{\prime} \\
c^{\prime} & d^{\prime}
\end{array}\right]\left[\begin{array}{cc}
1 & 0 \\
\frac{3^{n_{k}-1}}{2} & 3^{n_{k}}
\end{array}\right]=\left[\begin{array}{cc}
* & * \\
c^{\prime}+\frac{3^{n_{k}-1}}{2} d^{\prime} & 3^{n_{k}} d^{\prime}
\end{array}\right] ;
$$

since $k-1$ is even, we have $c^{\prime} \leq 3 d^{\prime}$. Therefore

$$
\gamma=c^{\prime}+\frac{3^{n_{k}}-1}{2} d^{\prime} \leq 3 d^{\prime}+3^{n_{k}} d^{\prime} \leq 2 \cdot 3^{n_{k}} d^{\prime}=2 \delta .
$$

We see that $\gamma \leq 3 \delta$ in both cases and the claim is proved.

The second inequality of (2.3) follows from

$$
M_{0}^{n_{1}} \cdots M_{\varepsilon}^{n_{k}} \leq\left[\begin{array}{ll}
3^{n_{1}} & 3^{n_{1}} \\
3^{n_{1}} & 3^{n_{1}}
\end{array}\right] \cdots\left[\begin{array}{ll}
3^{n_{k}} & 3^{n_{k}} \\
3^{n_{k}} & 3^{n_{k}}
\end{array}\right]=3^{n_{1}+\cdots+n_{k}}\left[\begin{array}{cc}
1 & 1 \\
1 & 1
\end{array}\right]^{k}
$$


and $[0,1]\left[\begin{array}{ll}1 & 1 \\ 1 & 1\end{array}\right]^{k}\left[\begin{array}{l}0 \\ 1\end{array}\right]=2^{k-1}$. For the first inequality of (2.3), we observe that

$$
M_{0}^{n_{1}} M_{2}^{n_{2}}=\left[\begin{array}{cc}
1 & 0 \\
\frac{3^{n_{1}}-1}{2} & 3^{n_{1}}
\end{array}\right]\left[\begin{array}{cc}
3^{n_{2}} & \frac{3^{n_{2}}-1}{2} \\
0 & 1
\end{array}\right]=\left[\begin{array}{cc}
* & * \\
* & \frac{\left(3^{n_{1}}-1\right)\left(3^{n_{2}}-1\right)}{4}+3^{n_{1}}
\end{array}\right] .
$$

Hence if $k$ is even, we have

$$
\begin{aligned}
\bar{c}\left(n_{1}, \cdots, n_{k}\right) & =[0,1]\left(M_{0}^{n_{1}} M_{2}^{n_{2}}\right) \cdots\left(M_{0}^{n_{k-1}} M_{2}^{n_{k}}\right)\left[\begin{array}{l}
0 \\
1
\end{array}\right] \\
& >\left(3^{n_{1}}-1\right)\left(3^{n_{2}}-1\right) \cdots\left(3^{n_{k}}-1\right) / 2^{k} \\
& \geq 3^{n_{1}+\cdots+n_{k}} / 3^{k} .
\end{aligned}
$$

If $k$ is odd, then note that $M_{0}^{n_{k}}\left[\begin{array}{l}0 \\ 1\end{array}\right]=3^{n_{k}}\left[\begin{array}{l}0 \\ 1\end{array}\right]$. Hence by using the previous result, we have

$$
\begin{aligned}
\bar{c}\left(n_{1}, \cdots, n_{k}\right) & =[0,1]\left(M_{0}^{n_{1}} M_{2}^{n_{2}}\right) \cdots\left(M_{0}^{n_{k-2}} M_{2}^{n_{k-1}}\right) M_{0}^{n_{k}}\left[\begin{array}{l}
0 \\
1
\end{array}\right] \\
& =\bar{c}\left(n_{1}, \cdots, n_{k-1}\right) \cdot 3^{n_{k}} \\
& \geq 3^{n_{1}+\cdots+n_{k}} / 3^{k-1} .
\end{aligned}
$$

Lemma 2.2. For a fixed $q \in \mathbb{R}$, let $\tilde{s}_{0}(q)=2, \tilde{s}_{1}(q)=2^{q}$ and

$$
\tilde{s}_{n}(q)=\sum_{J \in\{0,2\}^{n-1}}\left\|M_{J}\right\|^{q}, \quad n \geq 2 .
$$

Then for any fixed $\ell \in \mathbb{N}$, there exists $z_{\ell} \in\left(0,3^{-q}\right)$ such that

$$
\sum_{n_{1}, \cdots, n_{\ell} \geq 1} \bar{c}\left(n_{1}, \cdots, n_{\ell}\right)^{q}\left(z_{\ell}\right)^{n_{1}+\cdots+n_{\ell}}=1 .
$$

Moreover if let $R$ denote the radius of convergence of the series $\sum_{n \geq 2} \tilde{s}_{n}(q) x^{n-1}$, then $\sum_{n \geq 2} \tilde{s}_{n}(q) R^{n-1}=\infty$.

Proof. From (2.3), we have for any fixed $\ell$,

$$
c_{1}\left(\sum_{n \geq 1}\left(3^{q} x\right)^{n}\right)^{\ell} \leq \sum_{n_{1}, \cdots, n_{\ell} \geq 1} \bar{c}\left(n_{1}, \cdots, n_{\ell}\right)^{q} x^{n_{1}+\cdots+n_{\ell}} \leq c_{2}\left(\sum_{n \geq 1}\left(3^{q} x\right)^{n}\right)^{\ell},
$$

where $c_{1}=\min \left\{3^{-q \ell}, 2^{q \ell}\right\}$ and $c_{2}=\max \left\{3^{-q \ell}, 2^{q \ell}\right\}$. The existence of $z_{\ell}$ follows from the above inequalities. By the way,

$$
\sum_{n_{1}, \cdots, n_{\ell} \geq 1} \bar{c}\left(n_{1}, \cdots, n_{\ell}\right)^{q} x^{n_{1}+\cdots+n_{\ell}}<\infty, \quad \forall x \in\left(0,3^{-q}\right) .
$$

For the second part, we only give a proof in the case $q<0$; since the case for 
$q \geq 0$ has been considered in Part I. For each $\ell \in \mathbb{N}$, we write

$$
\begin{aligned}
\sum_{n \geq 2} \tilde{s}_{n}(q) x^{n-1} & =2 \sum_{k \geq 1} \sum_{n_{1}, \cdots, n_{k} \geq 1} c\left(n_{1}, \cdots, n_{k}\right)^{q} x^{n_{1}+\cdots+n_{k}} \\
& =2 \sum_{j=1}^{\ell} \sum_{n_{1}, \cdots, n_{j} \geq 1} c\left(n_{1}, \cdots, n_{j}\right)^{q} x^{n_{1}+\cdots+n_{j}} \\
& +2 \sum_{j=1}^{\ell} \sum_{k \geq 1} \sum_{n_{1}, \cdots, n_{k \ell+j} \geq 1} c\left(n_{1}, \cdots, n_{k \ell+j}\right)^{q} x^{n_{1}+\cdots+n_{k \ell+j}} \\
& :=2\left(I_{1}+I_{2}\right) .
\end{aligned}
$$

By using (2.1) for $q<0$, we have

$$
\begin{aligned}
I_{2} & \leq \sum_{j=1}^{\ell} \sum_{k \geq 1} \sum_{n_{1}, \cdots, n_{k \ell+j} \geq 1} \bar{c}\left(n_{1}, \cdots, n_{k \ell+j}\right)^{q} x^{n_{1}+\cdots+n_{k \ell+j}} \\
& \leq \sum_{j=1}^{\ell} \sum_{k \geq 1} \sum_{n_{1}, \cdots, n_{k \ell+j} \geq 1} \bar{c}\left(n_{1}, \cdots, n_{k \ell}\right)^{q} \bar{c}\left(n_{k \ell+1}, \cdots, n_{k \ell+j}\right)^{q} x^{n_{1}+\cdots+n_{k \ell+j}} \\
& \leq\left(\sum_{j=1}^{\ell} \sum_{n_{1}, \cdots, n_{j} \geq 1} \bar{c}\left(n_{1}, \cdots, n_{j}\right)^{q} x^{n_{1}+\cdots+n_{j}}\right) \cdot \sum_{k \geq 1}\left(\sum_{n_{1}, \cdots, n_{\ell} \geq 1} \bar{c}\left(n_{1}, \cdots, n_{\ell}\right)^{q} x^{n_{1}+\cdots+n_{\ell}}\right)^{k} .
\end{aligned}
$$

By (2.5) and the definition of $z_{\ell}$, we have $\sum_{n \geq 2} \tilde{s}_{n}(q) x^{n-1}$ converges on $\left(0, z_{\ell}\right)$. Thus $R \geq z_{\ell}$.

On the other hand, by (2.2), we have

$$
\sum_{n \geq 2} \tilde{s}_{n} x^{n-1} \geq 2 I_{1} \geq 2 \cdot 8^{q} \sum_{j=1}^{\ell} \sum_{n_{1}, \cdots, n_{j} \geq 1} \bar{c}\left(n_{1}, \cdots, n_{j}\right)^{q} x^{n_{1}+\cdots+n_{j}} .
$$

For a given integer $m \in \mathbb{N}$, let $\ell=2^{m}$. Then for $j=1,2, \ldots, 2^{m}$, by making use of (2.1), we have

$$
1=\sum_{n_{1}, \cdots, n_{\ell} \geq 1} \bar{c}\left(n_{1}, \cdots, n_{\ell}\right)^{q}\left(z_{\ell}\right)^{n_{1}+\cdots+n_{\ell}} \leq\left(\sum_{n_{1}, \cdots, n_{j} \geq 1} \bar{c}\left(n_{1}, \cdots, n_{j}\right)^{q}\left(z_{\ell}\right)^{n_{1}+\cdots+n_{j}}\right)^{\ell / j} .
$$

This implies that

$$
\sum_{n_{1}, \cdots, n_{j} \geq 1} \bar{c}\left(n_{1}, \cdots, n_{j}\right)^{q}\left(z_{\ell}\right)^{n_{1}+\cdots+n_{j}} \geq 1
$$

and

$$
\lim _{x \nearrow_{\ell}} \sum_{n \geq 2} \tilde{s}_{n}(q) x^{n-1} \geq 2 \cdot 8^{q}(m+1)
$$

Thus $\sum_{n \geq 2} \tilde{s}_{n} R^{n-1} \geq 8^{q}(m+1)$. Since $m$ is arbitrary, we have $\sum_{n \geq 2} \tilde{s}_{n} R^{n-1}=\infty$.

口

Now we can prove the main result of this section. 
THEOREM 2.3. There exists a unique real analytic function $r(q)>0$ satisfying

$$
\sum_{k=0}^{\infty} s_{k}(q) r(q)^{k+1}=1 \quad \text { and } \quad \sum_{k=1}^{\infty} k s_{k}(q) r(q)^{k}<\infty .
$$

$\left(s_{n}(q)\right.$ is defined in (1.2)). The $L^{q}$-spectrum of $\mu_{m}$ is independent of $m$ and is given by $\tilde{\tau}(q)=\log _{3} r(q)$.

Proof. We have proved the theorem for $q \geq-2$ in Part I. We will prove the theorem for $q<0$ here. It will be more convenient to use the $\tilde{s}_{n}(q)$ as in Lemma 2.2 than the $s_{n}(q)$. Note that in this case

$$
s_{n}(q)=3^{q} 8^{-(n+1) q} \tilde{s}_{n}(q), n=0,1, \cdots .
$$

If we let $\tilde{r}(q)=8^{-q} r(q)$, then (2.6) becomes

$$
3^{q} \sum_{k=0}^{\infty} \tilde{s}_{k}(q) \tilde{r}(q)^{k+1}=1 \quad \text { and } \quad \sum_{k=1}^{\infty} k \tilde{s}_{k}(q) \tilde{r}(q)^{k}<\infty
$$

and $\tilde{\tau}(q)=q \log _{3} 8+\log _{3} \tilde{r}(q)$. Let

$$
F(q, x):=3^{q} \sum_{n=0}^{\infty} \tilde{s}_{n}(q) x^{n+1} .
$$

For any fixed $q \in \mathbb{R}$, denote by $R(q)$ the radius of convergence of the series $\sum_{n=0}^{\infty} \tilde{s}_{n}(q) x^{n+1}$. Note that $F(q, 0)=0$ and $F(q, R(q))=\infty$ (Lemma 2.2); the continuity of $F(q, \cdot)$ implies that there exists $\tilde{r}(q)$ satisfies $(2.6)^{\prime}$. Also observe that for any fixed $q \in \mathbb{R}, F(q, \cdot)$ is a monotone function. Hence, $\tilde{r}(q)>0$ is unique.

The last part of the theorem was proved in Part I. $\mathrm{I}$

The following proposition describes the limit behavior of $\tilde{\tau}(q)$ at infinity.

Proposition 2.4. $\lim _{q \rightarrow \infty} \tilde{\tau}(q) / q=\underline{\alpha}, \lim _{q \rightarrow-\infty} \tilde{\tau}(q) / q=\tilde{\alpha}$, where

$$
\underline{\alpha}=\log _{3}(8 / 3), \quad \tilde{\alpha}=\log _{3}\left(8 / \sqrt{\lambda}_{1}\right),
$$

(see Theorem 1.2) where $\lambda_{1}=(7+\sqrt{13}) / 2$.

Proof. It was shown in $[\mathrm{LW}]$ that

$$
\tilde{\tau}(q)=-\lim _{n \rightarrow \infty}\left(\frac{1}{n} \log _{3} \sum_{|J|=n}\left(\mathbf{e}_{1}^{t} T_{J} \mathbf{1}\right)^{q}\right),
$$

where $\mathbf{e}_{1}^{t}=[0,1,0]$ and $\mathbf{1}^{t}=[1,1,1]$. For any $J=j_{1} \cdots j_{n} \in\{0,1,2\}^{n}$, we have

$$
\mathbf{e}_{1}^{t} T_{J} \mathbf{1} \leq \mathbf{1}^{t} T_{j_{1}} \cdots T_{j_{n}} \mathbf{1} \leq \frac{3}{8} \mathbf{1}^{t} T_{j_{2}} \cdots T_{j_{n}} \mathbf{1} \leq \cdots \leq 3\left(\frac{3}{8}\right)^{n} .
$$

Hence for $q>0$, we have

$$
\left(\frac{3}{8}\right)^{n q}=\left(\mathbf{e}_{1}^{t} T_{0}^{n} \mathbf{1}\right)^{q} \leq \sum_{|J|=n}\left(\mathbf{e}_{1}^{t} T_{J} \mathbf{1}\right)^{q} \leq 3^{n+q}\left(\frac{3}{8}\right)^{n q} .
$$


It follows that $\lim _{q \rightarrow+\infty} \tilde{\tau}(q) / q=\underline{\alpha}$.

To prove the second limit, making use of (A.3) and (A.4), we have

$$
C_{1}^{q}\left(\frac{\sqrt{\lambda_{1}}}{8}\right)^{n q} \leq \sum_{|J|=n}\left(\mathbf{e}_{1}^{t} T_{J} \mathbf{1}\right)^{q} \leq 3^{n} C_{2}^{q}\left(\frac{\sqrt{\lambda_{1}}}{8}\right)^{n q},
$$

for some constants $C_{1}, C_{2}>0$, and it follows that $\lim _{q \rightarrow-\infty} \tilde{\tau}(q) / q=\tilde{\alpha}$.

3. The restricted measure . In this section, we represent $\mu_{0}=\left.\mu\right|_{[1,2]}$ as a selfsimilar measure generated by an IFS with infinitely many non-overlapping similitudes.

Let $\Sigma=\bigcup_{n=1}^{\infty}\{0,1,2\}^{n}$ be the collection of all finite words over $\{0,1,2\}$. For $J=j_{1} \cdots j_{n}, J^{\prime}=j_{1}^{\prime} \cdots j_{m}^{\prime} \in \Sigma$, we say that $J$ and $J^{\prime}$ are incomparable if there exists some $k \leq \min \{n, m\}$ such that $j_{k} \neq j_{k}^{\prime}$. Let $\llbracket J \rrbracket$ be the subinterval $\left[\sum_{k=1}^{n} j_{k} 3^{-k}\right.$, $\left.\sum_{k=1}^{n} j_{k} 3^{-k}+3^{-n}\right] \subset[0,1]$ and let

$$
\varphi_{J}(x)=3^{-n}(x-1)+\sum_{k=1}^{n} j_{k} 3^{-k}+1 .
$$

Lemma 3.1. Let $J, J^{\prime} \in \Sigma$, then

(i) $\varphi_{J}([1,2])=\llbracket J \rrbracket+1 \subset[1,2]$;

(ii) $\varphi_{J J^{\prime}}=\varphi_{J} \circ \varphi_{J^{\prime}}$;

(iii) if $J$ and $J^{\prime}$ are incomparable, then $\mu_{0}\left(\varphi_{J}^{-1}\left(\llbracket J^{\prime} \rrbracket+1\right)\right)=0$.

Proof. It is direct to check (i) and (ii). For (iii), observe that

$$
\varphi_{J}\left(\varphi_{J}^{-1}\left(\llbracket J^{\prime} \rrbracket+1\right) \cap[1,2]\right)=\left(\llbracket J^{\prime} \rrbracket \cap \llbracket J \rrbracket\right)+1 .
$$

Since $J$ and $J^{\prime}$ are incomparable, there exists $k \leq \min \left\{|J|,\left|J^{\prime}\right|\right\}$ such that $j_{i}=j_{i}^{\prime}$ for $i<k$ and $j_{k} \neq j_{k}^{\prime}$. Thus $\llbracket J \rrbracket \cap \llbracket J^{\prime} \rrbracket \subset \llbracket j_{1} \cdots j_{k} \rrbracket \cap \llbracket j_{1}^{\prime} \cdots j_{k}^{\prime} \rrbracket$ which contains at most one point. Since $\mu_{0}$ does not have any point mass, (iii) follows. $\square$

Now let

$$
\Sigma_{0}=\{0,2\} \cup\left\{1 j_{1} \cdots j_{n} 1 \in \Sigma: n \geq 0, j_{k} \neq 1, k=1, \cdots, n\right\} .
$$

Let $T_{j}$ 's be defined as in Section 1. For each $J \in \Sigma_{0}$, we define $w_{J}$ as follows: $w_{0}=w_{2}=3 / 8$, and for the other $J, w_{J}=\mathbf{e}_{1}^{t} T_{J} \mathbf{e}_{1}$ where $\mathbf{e}_{1}^{t}=[0,1,0]$. By making use of [LW, Lemma 2], we have

$$
w_{J}=\left[p_{2}, 0, p_{1}\right] T_{j_{2} \cdots j_{n-1}} T_{1} \mathbf{e}_{1}=\left[p_{2}, p_{1}\right] \bar{T}_{j_{2} \cdots j_{n-1}}\left[\begin{array}{c}
p_{0} \\
p_{3}
\end{array}\right]=\frac{3}{8^{n}}\left\|M_{j_{2}} \cdots M_{j_{n-1}}\right\| .
$$

Observe that

$$
\left\|\left(M_{0}+M_{2}\right)^{n}\right\|=[1,1]\left[\begin{array}{ll}
4 & 1 \\
1 & 4
\end{array}\right]^{n}\left[\begin{array}{l}
1 \\
1
\end{array}\right]=2 \cdot 5^{n} .
$$

Hence

$$
\sum_{J \in \Sigma_{0}} w_{J}=2 \cdot \frac{3}{8}+\frac{3}{8^{2}} \sum_{n=0}^{\infty} \sum_{j_{1}, \cdots, j_{n}=0,2} \frac{1}{8^{n}}\left\|M_{j_{1}} \cdots M_{j_{n}}\right\|=\frac{3}{4}+\frac{3}{64} \sum_{n=0}^{\infty}\left\|\left(M_{0}+M_{2}\right)^{n}\right\| / 8^{n}=1 .
$$


THEOREM 3.2. The measure $\mu_{0}$ satisfies the following self-similar identity

$$
\mu_{0}=\sum_{J \in \Sigma_{0}} w_{J} \mu_{0} \circ \varphi_{J}^{-1}
$$

Furthermore $\left\{\varphi_{J}: J \in \Sigma_{0}\right\}$ has no overlap in the sense that $\varphi_{J}([1,2]) \subset[1,2]$, and $\varphi_{J}([1,2]) \cap \varphi_{J^{\prime}}([1,2])=\emptyset$ for any $J \neq J^{\prime}, J, J^{\prime} \in \Sigma_{0}$.

Proof. For the case $J=0,2, \varphi_{J}([1,2])$ are $\left[1, \frac{4}{3}\right]$ and $\left[\frac{5}{3}, 2\right]$ respectively. Let $C$ be the standard Cantor set in $\left[\frac{4}{3}, \frac{5}{3}\right]$, then for any $J \in \Sigma_{0} \backslash\{0,2\}, \llbracket J \rrbracket+1$ corresponds to the middle-third interval in the construction of the Cantor set. Since $\varphi_{J}([1,2])=\llbracket J \rrbracket+1$, the disjointness of the $\left\{\varphi_{J}([1,2]), J \in \Sigma_{0}\right\}$ implies the last part of the theorem.

Let $X=\bigcup_{J \in \Sigma_{0}}(\llbracket J \rrbracket+1)$. Then $X=[1,2] \backslash C$. From the vector-valued self-similar identity of $\boldsymbol{\mu}$ in (1.1), we see that $\mu_{0}$ is the middle entry of the expression. Hence

$$
\mu_{0}(\llbracket J \rrbracket+1)=\mathbf{e}_{1}^{t} T_{1 j_{2} \cdots j_{n-1} 1} \boldsymbol{\mu}([0,1])=\mathbf{e}_{1}^{t} T_{J} \mathbf{e}_{1} \mu([1,2])=w_{J} \mu_{0}([1,2]) .
$$

It follows that $\mu_{0}(X)=\sum_{J \in \Sigma_{0}} w_{J} \mu_{0}([1,2])=\mu_{0}([1,2])$, so that $\mu_{0}$ is concentrated in $X$. To prove the self-similar identity for $\mu_{0}$, it suffices to see that it holds on each $\llbracket J \rrbracket+1, J \in \Sigma_{0}$. Indeed for $A \subseteq \llbracket J \rrbracket+1$, let $B \subseteq[1,2]$ be such that $\varphi_{J}(B)=A$. It follows from Lemma 3.1(iii) that

$$
\sum_{J^{\prime} \in \Sigma_{0}} w_{J^{\prime}} \mu_{0} \circ \varphi_{J^{\prime}}^{-1}(A)=w_{J} \mu_{0} \circ \varphi_{J}^{-1}\left(\varphi_{J}(B)\right)=w_{J} \mu_{0}(B)=\mu_{0}(A)
$$

(the last equality follows from the same proof as (3.1)).

4. The modified multifractal formalism . In this section, we determine the dimension spectrum of $\mu$. Recall that the local dimensions $\alpha(x)$ has the range $E=$ $[\underline{\alpha}, \tilde{\alpha}] \cup\{\bar{\alpha}\}$ (see Theorem 1.2 and $[\mathrm{HL}]$ ). For each $\alpha \in E$, let

$$
K(\alpha)=\{x \in \operatorname{supp}(\mu): \alpha(x)=\alpha\} .
$$

Theorem 4.1. For any $\alpha \in(\underline{\alpha}, \tilde{\alpha})$,

$$
\operatorname{dim}_{H} K(\alpha)=\tilde{\tau}^{*}(\alpha),
$$

where $\tilde{\tau}(q)$ denotes the $L^{q}$-spectrum of $\mu_{0}$ (Theorem 2.3), i.e., $\tilde{\tau}^{*}(\alpha):=\inf \{\alpha q-\tilde{\tau}(q)$ : $q \in \mathbb{R}\}$.

We will prove the theorem for a more general measure than the $\mu_{0}$. Set $I=[0,1]$. Assume that $\left\{f_{i}\right\}_{i=1}^{\infty}$ is a family of similitudes on $\mathbb{R}$ with contraction ratios $\left\{r_{i}\right\}_{i=1}^{\infty}$. Furthermore we assume that $f_{i}(I) \subset I$ and $f_{i}(I) \cap f_{j}(I)=\emptyset$. Suppose there is a non-empty compact set $K \subset I$ such that

$$
K=\bigcup_{i=1}^{\infty} f_{i}(K) .
$$

For probability weights $\left\{w_{i}\right\}_{i=1}^{\infty}$, let $\bar{\mu}$ be a finite Borel measure on $K$ satisfying

$$
\bar{\mu}=\sum_{i=1}^{\infty} w_{i} \bar{\mu} \circ f_{i}^{-1} .
$$


Let $\mathbb{N}^{\infty}$ be the sequence space over $\mathbb{N}$ endowed with the product topology. For $J=$ $\left\{j_{i}\right\}_{i=1}^{\infty} \in \mathbb{N}^{\infty}$, let $J_{n}=j_{1} \ldots j_{n}$. Define $\pi: \mathbb{N}^{\infty} \mapsto I$ by

$$
\pi(J)=\lim _{n \rightarrow \infty} f_{J_{n}}(x),
$$

Note that the limit is independent of $x \in I$. It is clear that $K=\pi\left(\mathbb{N}^{\infty}\right)$.

Assume that for $q \in \mathbb{R}$, there exists $\eta(q)$ such that

$$
\sum_{i=1}^{\infty} w_{i}^{q} r_{i}^{-\eta(q)}=1
$$

Let $q \in \mathbb{R}$, let $\nu_{q}$ be the product measure with probability weight $\left\{w_{i}^{q} r_{i}^{-\eta(q)}: i \in \mathbb{N}\right\}$. It is well known that $\nu_{q}$ is an ergodic measure on $\mathbb{N}^{\infty}$. Define the measure $\mu_{q}$ on $I$ by

$$
\mu_{q}=\nu_{q} \circ \pi^{-1} .
$$

Lemma 4.2. Suppose $\sup _{1 \leq i<\infty}\left(\log w_{i} / \log r_{i}\right)<\infty$ and for each $q \in \mathbb{R}$, there exists $\eta(q)$ satisfies

$$
\sum_{i=1}^{\infty} w_{i}^{q} r_{i}^{-\eta(q)}=1 \quad \text { and } \quad-\sum_{i=1}^{\infty} w_{i}^{q} r_{i}^{-\eta(q)} \log r_{i}<\infty .
$$

Then there exists a Borel set $G_{q} \subset \mathbb{N}^{\infty}$ with $\nu_{q}\left(G_{q}\right)=1$ such that for any $J \in G_{q}$, the following holds:

$$
\begin{gathered}
\lim _{n \rightarrow \infty} \frac{1}{n} \log \mu_{q}\left(f_{J_{n}}(I)\right)=\sum_{i=1}^{\infty} w_{i}^{q} r_{i}^{-\eta(q)} \log \left(w_{i}^{q} r_{i}^{-\eta(q)}\right), \\
\lim _{n \rightarrow \infty} \frac{1}{n} \log \bar{\mu}\left(f_{J_{n}}(I)\right)=\sum_{i=1}^{\infty} w_{i}^{q} r_{i}^{-\eta(q)} \log w_{i} \\
\lim _{n \rightarrow \infty} \frac{1}{n} \log \left|f_{J_{n}}(I)\right|=\sum_{i=1}^{\infty} w_{i}^{q} r_{i}^{-\eta(q)} \log r_{i}
\end{gathered}
$$

where $\left|f_{J_{n}}(I)\right|$ denotes the length of the interval $f_{J_{n}}(I)$.

Proof. We use $\left[J_{n}\right]$ to denote the cylinder set in $\mathbb{N}^{\infty}$ with base $J_{n}$. Note that $f_{i}(I) \cap f_{j}(I)=\emptyset$ for any $i \neq j$, hence $\pi^{-1}\left(f_{J_{n}}(I)\right)=\left[J_{n}\right]$. By the definition of $\mu_{q}$ and $\nu_{q}$,

$$
\mu_{q}\left(f_{J_{n}}(I)\right)=\nu_{q}\left(\left[J_{n}\right]\right)=\prod_{i=1}^{n} w_{j_{i}}^{q} r_{j_{i}}^{-\eta(q)} .
$$

It follows that

$$
\frac{1}{n} \log \mu_{q}\left(f_{J_{n}}(I)\right)=\frac{1}{n} \sum_{i=1}^{n} \log \left(w_{j_{i}}^{q} r_{j_{i}}^{-\eta(q)}\right) .
$$


The limit (4.3) follows by applying the Birkhoff ergodic theorem to the i.i.d. random variables $\left\{X_{i}\right\}_{i=1}^{\infty}$ with values $\left\{\log \left(w_{i}^{q} r_{i}^{-\eta(q)}\right)\right\}_{i=1}^{\infty}$ and with probability $\left\{w_{i}^{q} r_{i}^{-\eta(q)}\right\}_{i=1}^{\infty}$. The other two limits follow from the same argument and

$$
\bar{\mu}\left(f_{J_{n}}(I)\right)=\left(\prod_{i=1}^{n} w_{j_{i}}\right) \bar{\mu}(I) \quad \text { and } \quad\left|f_{J_{n}}(I)\right|=\prod_{i=1}^{n} r_{j_{i}} .
$$

Let $\ell \in \mathbb{N}$ be fixed. For $J=\left(j_{i}\right)_{i=1}^{\infty} \in \mathbb{N}^{\infty}$, let $n_{1}=\min \left\{i \in \mathbb{N}: j_{i}=\ell\right\}$ and $n_{k+1}=\min \left\{i \in \mathbb{N}: i>n_{k}, j_{i}=\ell\right\}, k \geq 1$. We can choose the $G_{q}$ in the theorem satisfying in addition that each $J \in G_{q}$,

$$
\lim _{k \rightarrow \infty} \frac{n_{k+1}}{n_{k}}=1 .
$$

Indeed, by the Birkhoff ergodic theorem again, we have

$$
\lim _{n \rightarrow \infty} \frac{1}{n} \sum_{i=1}^{n} \chi_{\{\ell\}}\left(j_{i}\right)=w_{\ell}^{q} r_{\ell}^{-\eta(q)}, \quad \text { a.e. } \nu_{q}, \quad J \in \mathbb{N}^{\infty},
$$

where $\chi$ is the characteristic function. It follows that

$$
\lim _{k \rightarrow \infty} \frac{n_{k+1}}{n_{k}}=\lim _{k \rightarrow \infty}\left(\frac{1}{n_{k}} \sum_{i=1}^{n_{k}} \chi_{\{\ell\}}\left(j_{i}\right)\right) /\left(\frac{1}{n_{k+1}} \sum_{i=1}^{n_{k+1}} \chi_{\{\ell\}}\left(j_{i}\right)\right)=1 .
$$

For a measure $\nu$ on $\mathbb{R}^{d}$, denote

$$
K_{\nu}(\alpha):=\left\{x \in I: \lim _{\delta \rightarrow 0+} \frac{\log \nu([x-\delta, x+\delta])}{\log \delta}=\alpha\right\} .
$$

THEOREM 4.3. Let $\bar{\mu}$ be the self-similar measure defined as in (4.1). Suppose

$$
\sup _{1 \leq i<\infty}\left(\log w_{i} / \log r_{i}\right)<\infty
$$

and for each $q \in \mathbb{R}$ there exists $\eta(q)$ satisfying

$$
\sum_{i=1}^{\infty} w_{i}^{q} r_{i}^{-\eta(q)}=1 \quad \text { and } \quad-\sum_{i=1}^{\infty} w_{i}^{q} r_{i}^{-(\eta(q)+\epsilon)} \log r_{i}<\infty
$$

for some $\epsilon>0$. Then $\eta(q)$ is differentiable and

$$
\operatorname{dim}_{H} K_{\bar{\mu}}\left(\eta^{\prime}(q)\right)=\eta^{\prime}(q) q-\eta(q) .
$$

Proof. It is direct to show that under the hypothesis, $\eta(q)$ is differentiable. It is well known (cf., e.g., [LN, Theorem 4.1]) that

$$
\operatorname{dim}_{H} K_{\bar{\mu}}\left(\eta^{\prime}(q)\right) \leq \eta^{\prime}(q) q-\eta(q) .
$$

To prove the reverse inequality, we consider the set $G_{q}$ in Lemma 4.2. First we show that for each $J \in G_{q}$ and for $x_{J}=\pi(J)$, the following identity holds:

$$
\lim _{\delta \rightarrow 0+} \frac{\log \bar{\mu}\left(\left[x_{J}-\delta, x_{J}+\delta\right]\right)}{\log \delta}=\eta^{\prime}(q) .
$$


To see this, fix $J=\left(j_{i}\right)_{i=1}^{\infty} \in G_{q}$ and let $\left\{n_{k}\right\}_{k=1}^{\infty}$ be the sequence in (4.6). For a sufficient small $\delta>0$, there exists $k \in \mathbb{N}$ such that $r_{J_{n_{k+1}}} \leq \delta<r_{J_{n_{k}}}$, where $r_{j_{1} \ldots j_{n}}:=r_{j_{1}} \ldots r_{j_{n}}$. Then $f_{J_{n_{k+1}}}(I) \subset\left[x_{J}-\delta, x_{J}+\delta\right]$. It follows from (4.4) and (4.5) that

$$
\varlimsup_{\delta \rightarrow 0} \frac{\log \bar{\mu}\left(\left[x_{J}-\delta, x_{J}+\delta\right]\right)}{\log \delta} \leq \varlimsup_{\delta \rightarrow 0} \frac{\log \bar{\mu}\left(f_{J_{n_{k+1}}}(I)\right)}{\log \left|f_{J_{n_{k}}}(I)\right|} \leq \frac{\sum_{i=1}^{\infty} w_{i}^{q} r_{i}^{-\eta(q)} \log w_{i}}{\sum_{i=1}^{\infty} w_{i}^{q} r_{i}^{-\eta(q)} \log r_{i}}=\eta^{\prime}(q) .
$$

To prove the other inequality, we assume without loss of generality that $f_{2}(I)$ is in between $f_{1}(I)$ and $f_{3}(I)$. Let $\left\{n_{k}\right\}_{k=1}^{\infty}$ be given as in (4.6) with $\ell=2$. Let us denote $\bar{J}=J_{n_{k+1}-1}$ for short, then $\bar{J} 2=J_{n_{k+1}}$,

$$
x_{J} \in f_{\bar{J} 2}(I) \quad \text { and } \quad \bigcup_{i=1}^{3} f_{\bar{J} i}(I) \subset f_{J_{n_{k}}}(I) .
$$

If we take $\delta=r_{\bar{J}} \cdot \min \left\{r_{1}, r_{3}\right\}$, then $\delta>c r_{J_{n_{k+1}}}=c\left|f_{J_{n_{k+1}}}(I)\right|$ for some $c>0$, and $\left[x_{J}-\delta, x_{J}+\delta\right] \subset f_{J_{n_{k}}}(I)$. It follows that

$$
\frac{\log \bar{\mu}\left(\left[x_{J}-\delta, x_{J}+\delta\right]\right)}{\log \delta} \geq \frac{\log \bar{\mu}\left(f_{J_{n_{k}}}(I)\right)}{\log c\left|f_{J_{n_{k+1}}}(I)\right|} .
$$

Making use of (4.4) and (4.5) again, we have

$$
\varliminf_{\delta \rightarrow 0} \frac{\left.\log \bar{\mu}\left(x_{J}-\delta, x_{J}+\delta\right]\right)}{\log \delta} \geq \eta^{\prime}(q) .
$$

This completes the proof of (4.7).

If we replace $\bar{\mu}$ with $\mu_{q}$ and use (4.2) in the above arguments, then

$$
\lim _{\delta \rightarrow 0} \frac{\log \mu_{q}\left(\left[x_{J}-\delta, x_{J}+\delta\right]\right)}{\log \delta}=\frac{\sum_{i=1}^{\infty} w_{i}^{q} r_{i}^{-\eta(q)} \log \left(w_{i}^{q} r_{i}^{-\eta(q)}\right)}{\sum_{i=1}^{\infty} w_{i}^{q} r_{i}^{-\eta(q)} \log r_{i}}=\eta^{\prime}(q) q-\eta(q)
$$

for all $J \in G_{q}$. It follows from $[\mathrm{Y}]$ that

$$
\operatorname{dim}_{H} \mu_{q}=\eta^{\prime}(q) q-\eta(q) .
$$

Therefore $\operatorname{dim}_{H} \pi\left(G_{q}\right) \geq \eta^{\prime}(q) q-\eta(q)$. Observe that (4.7) implies that $\pi\left(G_{q}\right) \subset$ $K_{\bar{\mu}}\left(\eta^{\prime}(q)\right)$. This implies the theorem. that

Proof of Theorem 4.1. Observe that $K(\alpha) \subset\left(\sum_{m=0}^{\infty} K_{\mu_{m}}(\alpha)\right) \cup\{0,3\}$. It follows

$$
\operatorname{dim}_{H} K(\alpha) \leq \max _{m} \operatorname{dim}_{H} K_{\mu_{m}}(\alpha)=\operatorname{dim}_{H} K_{\mu_{0}}(\alpha) \leq \tilde{\tau}^{*}(\alpha)
$$

To prove the reverse inequality, we apply Theorem 4.3 to the family $\left\{\varphi_{J}: J \in\right.$ $\left.\Sigma_{0}\right\}$ defined in Section 3; the conditions of Theorem 4.3 hold for $\eta(q)=\tilde{\tau}(q)$. By Proposition 2.4, $(\underline{\alpha}, \tilde{\alpha})=\left\{\alpha \in \mathbb{R}: \alpha=\tilde{\tau}^{\prime}(q)\right.$ for some $\left.q \in \mathbb{R}\right\}$. Hence if we take $\alpha=\tilde{\tau}^{\prime}(q)$, then $\operatorname{dim}_{H} K(\alpha) \geq \operatorname{dim}_{H} K_{\mu_{0}}(\alpha) \geq \tilde{\tau}^{*}(\alpha)$.

Appendix A. Local dimensions. The proof of Theorem 1.2 in [HL] is based on some complicated combinatorial analysis on the multiple representation of 
$\sum_{i=0}^{\infty} \epsilon_{i} 3^{-i}, \epsilon_{i}=0,1,2,3$. In the following we give two other proofs: one is a consequence of Theorem 4.1 and another one is based on the direct estimate of the product of matrices.

For $x \in \operatorname{supp} \mu=[0,3]$, define

$$
\bar{\alpha}(x)=\limsup _{\delta \rightarrow 0} \frac{\log \mu[x-\delta, x+\delta]}{\log \delta}
$$

and define $\underline{\alpha}(x)$ similarly by taking the lower limit.

Lemma A.1. Let $J=j_{1} j_{2} \cdots \in\{0,1,2\}^{\mathbb{N}}$. For a given $j_{0} \in\{0,1,2\}$, set $x=\sum_{k=0}^{\infty} \frac{j_{k}}{3^{k}}$. Then

$$
\bar{\alpha}(x)=-\limsup _{n \rightarrow \infty} \frac{1}{n}\left(\log _{3} \mathbf{e}_{j_{0}}^{t} T_{j_{1} \cdots j_{n}} \mathbf{1}\right) .
$$

Similar equality holds for $\underline{\alpha}(x)$ by taking the lower limit respectively.

Proof. Denote $J_{n}=j_{1} \cdots j_{n}$. Then $x \in \llbracket J_{n} \rrbracket+j_{0}$. By (1.1) (see also [LW], Proposition 2.1), we have

$$
\mu\left(\llbracket J_{n} \rrbracket+j_{0}\right)=\mathbf{e}_{j_{0}}^{t} T_{j_{1} \cdots j_{n}} \mathbf{a},
$$

where $\mathbf{a}=\boldsymbol{\mu}([0,1])$. To prove the lemma, we only need to prove that there exist $C_{1}, C_{2}>0$ such that

$$
C_{1} \mu\left(\llbracket J_{n} \rrbracket+j_{0}\right) \leq \mu\left(\llbracket J_{n} \rrbracket+j_{0}-3^{-n}\right) \leq C_{2} \mu\left(\llbracket J_{n} \rrbracket+j_{0}\right), \quad \forall n \in \mathbb{N} .
$$

Indeed, the above inequalities can be checked directly. Here we only consider the case $J_{n}=j_{1} \ldots j_{n}$ with $j_{t}=0$ for $t=k+1, \ldots, n$ while $j_{k}>0$. In this case,

$$
\llbracket J_{n} \rrbracket+j_{0}-3^{-n}=\llbracket j_{1} \cdots j_{k-1}\left(j_{k}-1\right) 2 \cdots 2 \rrbracket+j_{0} .
$$

It follows that

$$
\mu\left(\llbracket J_{n} \rrbracket+i\right)=\mathbf{e}_{i}^{t} T_{j_{1} \cdots j_{k-1}} T_{j_{k}} T_{0}^{n-k} \mathbf{a},
$$

and

$$
\mu\left(\llbracket J_{n} \rrbracket+i-3^{-n}\right)=\mathbf{e}_{i}^{t} T_{j_{1} \cdots j_{k-1}} T_{j_{k}-1} T_{2}^{n-k} \mathbf{a} .
$$

A direct calculation for $T_{0}^{\ell}, T_{2}^{\ell}$ shows that

$$
T_{j_{k}} T_{0}^{n-k} \mathbf{a}, \quad T_{j_{k}-1} T_{2}^{n-k} \mathbf{a} \approx\left(\frac{3}{8}\right)^{n-k} \mathbf{a}
$$

for $j_{k}=1$ or 2 . Thus $\mu\left(\llbracket J_{n} \rrbracket+i-3^{-n}\right) \approx \mu\left(\llbracket J_{n} \rrbracket+i\right)(i=0,1,2)$, which implies (A.1).

Lemma A.2. Let $\mathbf{e}_{1}^{t}=[0,1,0]$ and $\mathbf{e}_{3}^{t}=[1,0,1]$. Then $T_{1}=\frac{1}{8}\left(\mathbf{e}_{3} \mathbf{e}_{1}^{t}+3 \mathbf{e}_{1} \mathbf{e}_{3}^{t}\right)$. Furthermore for any $n \in \mathbb{N}$ and $J \in\{0,2\}^{n}$, we have

$$
\mathbf{e}_{1}^{t} T_{J} \mathbf{e}_{3}=\mathbf{e}_{3}^{t} T_{J} \mathbf{e}_{1}=0 ; \quad \mathbf{e}_{1}^{t} T_{J} \mathbf{e}_{1}=\mathbf{e}_{1}^{t} T_{J} \mathbf{1}=\left(\frac{3}{8}\right)^{n} ; \quad \mathbf{e}_{3}^{t} T_{J} \mathbf{e}_{3}=\mathbf{e}_{3}^{t} T_{J} \mathbf{1}=\left(\frac{1}{8}\right)^{n}\left\|M_{J}\right\| .
$$


Proof. We only need to observe that for $J \in\{0,2\}^{n}, T_{J}$ has the form $\left[\begin{array}{ccc}\alpha_{1} & 0 & \alpha_{2} \\ 0 & \alpha_{3} & 0 \\ \alpha_{4} & 0 & \alpha_{5}\end{array}\right]$. The rest of the proof is direct.

Proof of Theorem 1.2. It follows from Proposition 2.4 and Theorem 4.1 that the domain of $\tilde{\tau}^{*}(\alpha)$ is $(\underline{\alpha}, \tilde{\alpha})$, and all the local dimension $\alpha$ of $\mu$ in $(\underline{\alpha}, \tilde{\alpha})$ are attainable for some $x \in(0,3)$.

For the three specific $\alpha$ 's, it is a direct check that for $x=0,3$,

$$
\alpha(x)=\log _{3} 8=\bar{\alpha} .
$$

If we take $x=1+\sum_{k=1}^{\infty} j_{k} 3^{-k}, j_{k}=0$ or 2 , then Lemma A.1 and Lemma A.2 implies that

$$
\alpha(x)=\log _{3}(8 / 3)=\underline{\alpha} .
$$

For $\tilde{\alpha}$, recall that we have proved in [LW, Lemma 3.2] that

$$
2\left(\lambda_{1}\right)^{n / 2} \leq\left\|M_{J_{n}^{0}}\right\| \leq 5\left(\lambda_{1}\right)^{n / 2}, \quad\left\|M_{J_{n}^{0}}\right\|=\left\|M_{J_{n}^{2}}\right\|=\min \left\{\left\|M_{J}\right\|:|J|=n\right\},
$$

where $J_{n}^{0}=\varepsilon \cdots 2020, J_{n}^{2}=(2-\varepsilon) \cdots 0202(\varepsilon=0$ or 2 according to $n$ is odd or even), the alternative sequence of 0 and 2 with length $n$.

Let $J=j_{0} j_{1} \cdots=11020202 \cdots$ and $x=\sum_{k=0}^{\infty} j_{k} 3^{-k}=17 / 12$, then by Lemma A.2 and (A.2), we have

$$
\mathbf{e}_{j_{0}}^{t} T_{j_{1} \cdots j_{n}} \mathbf{1}=3\left(\frac{1}{8}\right)^{n}\left\|M_{j_{2} \cdots j_{n}}\right\| \approx\left(\frac{\sqrt{\lambda_{1}}}{8}\right)^{n} .
$$

Lemma A.1 implies that

$$
\alpha(x)=\log _{3}\left(8 / \sqrt{\lambda_{1}}\right)=\tilde{\alpha} .
$$

$\square$

We can also prove Theorem 1.2 by a direct use of the product of matrices instead of going through Theorem 4.1 on the $L^{q}$-spectrum and the Legendre transform.

Lemma A.3. For any $x \in(0,3)$, we have $\underline{\alpha} \leq \underline{\alpha}(x) \leq \bar{\alpha}(x) \leq \tilde{\alpha}$.

Proof. For $x \in(0,3)$, we can write $x=\sum_{k=0}^{\infty} j_{k} 3^{-k}, j_{k} \in\{0,1,2\}$ and $j_{0}, j_{1}, \cdots$ are not all 0 or all 2 .

It is easy to verify that for any $i \in\{0,1,2\}, j \in\{0,1,2,3\}$, there exists some $k \in\{0,1,2,3\}$ such that $\mathbf{e}_{j}^{t} T_{i} \leq \frac{3}{8} \mathbf{e}_{k}$, where $\mathbf{e}_{3}^{t}=[1,0,1]$. This implies that

$$
\mathbf{e}_{j_{0}}^{t} T_{j_{1} \cdots j_{n}} \mathbf{1} \leq 2\left(\frac{3}{8}\right)^{n}
$$

By Lemma A.1, it follows that $\underline{\alpha}(x) \geq \log _{3} 8 / 3=\underline{\alpha}$.

To prove $\bar{\alpha}(x) \leq \tilde{\alpha}$, we claim that for $J \in\{0,1,2\}^{n}$,

$$
\mathbf{e}^{t} T_{J} \mathbf{1} \geq C_{1}\left(\frac{\sqrt{\lambda_{1}}}{8}\right)^{n}
$$


where $\mathbf{e}=\mathbf{e}_{1}$ or $\mathbf{e}_{3}$. Indeed let $N(J)$ be the total number of entries of $J$ that equals to 1 . If $N(J)=2 k$ is even, we write $J=J_{1} 1 J_{2} 1 \cdots J_{2 k} 1 J_{2 k+1}, J_{i} \in \cup_{l=0}^{\infty}\{0,2\}^{l}$. By repeated use of Lemma A.2, we have

$$
\begin{aligned}
& \mathbf{e}_{1}^{t} T_{J} \mathbf{1}=\left(\frac{3}{8}\right)^{\left|J_{2 k+1}\right|} \prod_{i=1}^{k}\left(\frac{3}{8}\right)^{1+\left|J_{2 i-1}\right|}\left(\frac{1}{8}\right)^{1+\left|J_{2 i}\right|}\left\|M_{J_{2 i}}\right\|, \\
& \mathbf{e}_{3}^{t} T_{J} \mathbf{1}=\left(\frac{1}{8}\right)^{\left|J_{2 k+1}\right|}\left\|M_{J_{2 k+1}}\right\| \prod_{i=1}^{k}\left(\frac{1}{8}\right)^{1+\left|J_{2 i-1}\right|}\left(\frac{3}{8}\right)^{1+\left|J_{2 i}\right|}\left\|M_{J_{2 i-1}}\right\| .
\end{aligned}
$$

By making use of (A.2) and noting that $\lambda_{1}<6$, we can show that $\mathbf{e}^{t} T_{J} \mathbf{1}>\left(\frac{\sqrt{\lambda_{1}}}{8}\right)^{|J|}$.

If $N(J)=2 k+1$ is odd, we write $J=J_{1} 1 J_{2}$, where $J_{1} \in \cup_{l=0}^{\infty}\{0,2\}^{l}$ and $N\left(J_{2}\right)=2 k$ is even. Applying Lemma A.2 and the above estimation to $J_{2}$ yields the claim.

Now to complete the proof of the lemma, note that $j_{0}, j_{1}, \cdots$ are not all 0 or all 2. Thus there exist some $k \geq 0$ and $C_{2}>0$ such that

$$
\mathbf{e}_{j_{0}}^{t} T_{j_{1} \cdots j_{k}} \geq C_{2} \mathbf{e}
$$

where $\mathbf{e}=\mathbf{e}_{1}$ or $\mathbf{e}_{3}$. By the claim, for any $n \geq k$, we have

$$
\mathbf{e}_{j_{0}}^{t} T_{j_{1} \cdots j_{n}} \mathbf{1} \geq C_{2} \mathbf{e} T_{j_{k+1} \cdots j_{n}} \geq C_{1} C_{2}\left(\frac{\sqrt{\lambda_{1}}}{8}\right)^{n-k} .
$$

Lemma A.1 implies that

$$
\bar{\alpha}(x)=-\varlimsup_{n \rightarrow \infty} \frac{1}{n}\left(\log _{3} \mathbf{e}_{j_{0}}^{t} T_{j_{1} \cdots j_{n}} \mathbf{1}\right) \leq \log _{3}\left(8 / \sqrt{\lambda_{1}}\right)=\tilde{\alpha} .
$$

口

Lemma A.4. For any $0<\theta<1$, there exist integer sequences $\left\{x_{k}\right\}_{k=1}^{\infty}$ and $\left\{y_{k}\right\}_{k=1}^{\infty}$ such that

$$
\lim _{k \rightarrow \infty} \frac{x_{k}}{\sum_{i=1}^{k}\left(x_{i}+y_{i}\right)}=\lim _{k \rightarrow \infty} \frac{y_{k}}{\sum_{i=1}^{k}\left(x_{i}+y_{i}\right)}=\lim _{k \rightarrow \infty} \frac{k}{\sum_{i=1}^{k}\left(x_{i}+y_{i}\right)}=0,
$$

and

$$
\lim _{k \rightarrow \infty} \frac{\sum_{i=1}^{k} x_{i}}{\sum_{i=1}^{k}\left(x_{i}+y_{i}\right)}=\theta, \quad \lim _{k \rightarrow \infty} \frac{\sum_{i=1}^{k} y_{i}}{\sum_{i=1}^{k}\left(x_{i}+y_{i}\right)}=1-\theta .
$$

Proof. Let $x_{k}=[\theta k]$ and $y_{k}=k-x_{k}$, where $[x]$ is the integral part of $x$. Then $\left\{x_{k}\right\}_{k=1}^{\infty}$ and $\left\{y_{k}\right\}_{k=1}^{\infty}$ satisfy all the conditions. $\square$

Another proof of Theorem 1.2. We have shown that the three specific values of $\alpha$ 's are attainable. It remains to consider the case for $\underline{\alpha}<\alpha<\tilde{\alpha}$. We write $\alpha=\theta \underline{\alpha}+(1-\theta) \tilde{\alpha}$. Let $\left\{x_{n}\right\}_{n=1}^{\infty}$ and $\left\{y_{n}\right\}_{n=1}^{\infty}$ be the sequences in Lemma A.4. Let $J_{n}=\underbrace{00 \cdots 0}_{x_{n}} 1 \underbrace{2020 \cdots}_{y_{n}} 1$ and put these segments together as $J=1 j_{1} j_{2} \cdots:=$ $1 J_{1} J_{2} \ldots$. A similar calculation as Lemma A.3 yields

$$
\mathbf{e}_{1}^{t} T_{J_{1} J_{2} \cdots J_{k}} \mathbf{1}=\prod_{i=1}^{k}\left(\frac{3}{8}\right)^{x_{i}+1}\left(\frac{1}{8}\right)^{y_{i}+1}\left\|M_{I\left(y_{n}\right)}\right\|,
$$


where $I\left(y_{n}\right)=\underbrace{2020 \cdots}_{y_{n}}$, the alternative sequence of 2 and 0 with length $y_{n}$. By making use of (A.2), we know that there exists $\frac{6}{64}<C(k)<\frac{15}{64}$ such that

$$
\mathbf{e}_{1}^{t} T_{J_{1} J_{2} \ldots J_{k}} \mathbf{1}=C(k)^{k} \prod_{i=1}^{k}\left(\frac{3}{8}\right)^{x_{i}}\left(\frac{\sqrt{\lambda_{1}}}{8}\right)^{y_{i}} .
$$

For any $n \in \mathbb{N}$, there exists $k \in \mathbb{N}$ such that $\sum_{i=1}^{k-1}\left|J_{i}\right|<n \leq \sum_{i=1}^{k}\left|J_{i}\right|$, i.e.,

$$
2(k-1)+\sum_{i=1}^{k-1}\left(x_{i}+y_{i}\right)<n \leq 2 k+\sum_{i=1}^{k}\left(x_{i}+y_{i}\right) .
$$

It follows that

$$
\mathbf{e}_{1}^{t} T_{J_{1} J_{2} \cdots J_{k}} \mathbf{1} \leq \mathbf{e}_{1}^{t} T_{j_{1} j_{2} \cdots j_{n}} \mathbf{1} \leq \mathbf{e}_{1}^{t} T_{J_{1} J_{2} \cdots J_{k-1}} \mathbf{1} .
$$

By taking logarithm, together with (A.5) and the special properties of $\left\{x_{n}\right\}_{n=1}^{\infty},\left\{y_{n}\right\}_{n=1}^{\infty}$ in Lemma A.4, we conclude that

$$
-\lim _{n \rightarrow \infty} \frac{1}{n}\left(\log _{3} \mathbf{e}_{1}^{t} T_{j_{1} \cdots j_{n}} \mathbf{1}\right)=\left(\theta \log _{3}(8 / 3)+(1-\theta) \log _{3}\left(8 / \sqrt{\lambda_{1}}\right)\right)=\alpha,
$$

this completes the proof.

Recently Shmerkin $[\mathrm{S}]$ independently considered the multifractal structure of the 3 -fold convolution of the Cantor measure and the extension from a different approach. Testud $[\mathrm{T}]$ found some interesting phase transition behaviors for another class of selfsimilar measures.

\section{REFERENCES}

[F] D.J. FEnG, The limited Rademacher functions and Bernoulli convolutions associated with Pisot numbers, Adv. Math., 195 (2005), pp. 24-101.

[FO] D.J. Feng And E. Olivier, Multifractal Analysis of the Weak Gibbs Measures and phase transition-application to some Bernoulli convolutions, Ergodic Theory \& Dynamical System, 23 (2003), pp. 1751-1784.

[HL] T.Y. HU AND K.S. LAU, Multifractal structure of convolution of the Cantor measure, Adv. Appl. Math., 27 (2001), pp. 1-16.

[LN] K.S. LAU AND S.M. NGAI, Multifractal measures and a weak separation condition, Adv. Math., 141 (1999), pp. 45-96.

[LW] K.S. LaU AND X.Y. WAnG, Some exceptional phenomena in multifractal formalism: Part I, Asian J. Math., 9 (2005), pp. 275-294.

[RM] R. Riedi And B. Mandelbrot, Multifractal formalism for infinite multinomial measures, Adv. Appl. Math., 16 (1995), pp. 132-150.

[S] P. Shmerkin, A modified multifractal formalism for a class of self-similar measures with overlaps, preprint.

[T] B. Testud, Etude d'une classe de mesures autosimilaries: calculs de dimensions et analyse multifractale, Ph. D. Thesis. (2005).

[Y] L.S. Young, Dimension, entropies and Liapunov exponents, Ergod. Theory Dyn. Sys., (1982), pp. 109-124. 